\title{
Correlación y conversión entre valores de colinesterasa eritrocitaria medida con las técnicas de Michel y EQM ${ }^{\circledR}$
}

\author{
Jaime Carmona-Fonseca \\ Grupo de Malaria, Facultad de Medicina, Universidad de Antioquia, Medellín, Colombia.
}

Introducción. Las técnicas para medir colinesterasa eritrocitaria son muchas y es útil tener un modelo matemático que permita interconvertir valores.

Objetivo. A partir de datos autóctonos de valores de referencia de actividad colinesterásica medida por las técnicas de Michel y EQM ${ }^{\circledast}$ hallar una ecuación para transformar unas unidades en otras.

Materiales y métodos. Diseño descriptivo, transversal y prospectivo, con sendas muestras representativas de poblaciones laborales adultas, de 18 a 75 años de edad, no expuestas a plaguicidas inhibidores de colinesterasa, vinculadas al Seguro Social y situadas en el valle de Aburrá y en el cercano oriente antioqueño (Antioquia, Colombia).

Resultados. En las 827 personas, las pruebas eritrocitarias cuantitativas (Michel y EQM ${ }^{\circledast}$ ) tienen coeficiente «r» entre 0,666 y coeficiente $\mathrm{R}^{2}$ de $44 \%$. La correlación es mayor en Aburrá que en Oriente. El modelo lineal en los 827 sujetos es: $\mathrm{EQM}^{\circledR}$ en U/g oxihemoglobina $=9,57509$ $\mathrm{U} / \mathrm{g}$ oxihemoglobina $+29,7914$ (Michel en deltas de $\mathrm{pH} / \mathrm{hora}$ ), Michel en deltas de $\mathrm{pH} / \mathrm{hora}=$ 0,33120 deltas de $\mathrm{pH} /$ hora $+0,01487\left(\mathrm{EQM}^{\circledast}\right.$ en $\mathrm{U} / \mathrm{g}$ oxihemoglobina). Tanto las intersecciones (coeficiente a) como las pendientes (coeficiente b) son significativas en el modelo. En las ecuaciones ajustadas, al excluir doce datos extremos (1,5\% de 827), el coeficiente $r$ sube de 0,666 a más de 0,718 y ellas son: EQM U/g oxihemoglobina $=8,18840 \mathrm{U} / \mathrm{g}$ oxihemoglobina + 31,3920 (Michel deltas de $\mathrm{pH} /$ hora), Michel deltas de $\mathrm{pH} / \mathrm{hora}=0,292510$ deltas de $\mathrm{pH} / \mathrm{hora}$ $+0,016101$ (EQM U/g oxihemoglobina).

Conclusión. Este sistema de ecuaciones lineales permitirá convertir unidades Michel (delta $\mathrm{PH} /$ hora) en unidades EQM (U/g oxihemoglobina) y viceversa, lo cual facilitará el trabajo en ambientes clínicos y epidemiológicos.

Palabras clave: acetilcolina, acetilcolinesterasa, plaguicidas, inhibidores de la colinesterasa, vigilancia epidemiológica.

Correlation and interconversion between erythrocyte cholinesterase values obtained by the Michel and the $\mathrm{EQM}^{\circledR}$ techniques

Introduction. Several techniques are available to measure red cell cholinesterase; therefore, evaluations with several methods provide a measure of concordance.

Objective. An equation was formulated to transform native data of reference values to reference units of cholinesterase activity as measured by Michel and EQM ${ }^{\circledR}$ tests.

Materials and methods. The experimental design was descriptive, transversal and prospective. The group sampled was a representative adult working population, aged 18-75, without previous exposure to cholinesterase inhibitors pesticides. The individuals were affiliated to the Social Security System and resided in Valle de Aburrá and Cercano Oriente Antioqueño (Antioquia Province, northwestern Colombia).

Results. Of 827 individuals, quantitative erythrocytes (Michel y $\mathrm{EQM}^{\circledR}$ ) tests exhibited "r" coefficients between 0.67 and $\mathrm{R}^{2}$ coefficient of $44 \%$., This indicated that one test explained the results in other test in $44 \%$ of the cases. The corelation was higher in Aburrá than in Oriente. The linear model for the 827 individuals was as follows: EQM U/g oxy-hemoglobin $=9.575 \mathrm{U} /$ g oxy-hemoglobin +29.791 (Michel delta $\mathrm{pH} /$ hour). Michel delta $\mathrm{pH} / \mathrm{hr}=0.3312$ delta $\mathrm{pH} / \mathrm{hour}$ + 0.0149 (EQM U/g oxy-hemoglobin), where $\mathrm{EQM}^{\circledR}$ was expressed in $\mathrm{U} / \mathrm{g}$ oxy-hemoglobin and 


\begin{abstract}
Michel $\mathrm{pH}$ change/hr. Inter-sections (coefficient a) and inclines (coefficient b) were significant in this model.

In the adjusted equations, after exclusion of 12 extreme data (1.5\% of 827$)$, the $r$ coefficient increased from 0.67 to 0.72 The adjusted equations were as follows: EQM U/g oxy-hemoglobin $=8.1884 \mathrm{U} / \mathrm{g}$ oxy-hemoglobin +31.3920 (Michel delta $\mathrm{pH} /$ hour); Michel delta $\mathrm{pH} / \mathrm{hr}=0.2925$ delta $\mathrm{pH} / \mathrm{hr}+0.0161$ (EQM U/g oxy-hemoglobin).

Conclusion. This system of linear equations permitted the transformation of Michel (delta $\mathrm{PH} /$ hr) units to EQM (U/g oxy-hemoglobin) units and vice versa. This will facilitate data comparisons by clinicians and epidemiologists who are using these methods of cholinesterase measurement.
\end{abstract}

Key words: acetylcholine, acetylcholinesterase, pesticides, cholinesterase inhibitors, epidemiological surveillance.

Las actividades sanitarias de control de insectos y, sobre todo, las prácticas agrícolas en la mayor parte de los países del mundo hacen uso intenso de muchos plaguicidas químicos y, aunque se usen en forma correcta, los plaguicidas producen efectos nocivos agudos y crónicos en la salud humana y al ambiente; los plaguicidas inhibidores de las colinesterasas son de los más empleados (1-3). Tanto el uso de los plaguicidas como las intoxicaciones que ocasionan obligan a mejorar los programas de vigilancia epidemiológica para las personas expuestas y la atención médica para las intoxicadas.

La acetilcolina es un mediador del impulso nervioso $(4,5)$ y las enzimas que producen su hidrólisis y la de otros ésteres de la colina se llaman colinesterasas, las cuales son de dos tipos (4-6): a) colinesterasa eritrocitaria, verdadera, específica o de tipo e, que se encuentra exclusivamente en las neuronas, en las sinapsis ganglionares de la estructura neuromuscular del organismo y en los eritrocitos; b) colinesterasa plasmática, sérica, seudocolinesterasa, colinesterasa inespecífica, butirilcolinesterasa, o de tipo s, la cual está presente en casi todos los tejidos (principalmente en hígado) y en el plasma, pero en poca concentración en el sistema nervioso central y periférico.

El indicador biológico de exposición humana comprende cualquier sustancia o subproducto de

\footnotetext{
Correspondencia:

Jaime Carmona-Fonseca, Grupo de Malaria, Facultad de Medicina, Universidad de Antioquia, Medellín, Colombia. Calle 62 No.52-59, laboratorio 610, Medellín, Colombia. Telfax: (574) 2106487

jaimecarmonaf@hotmail.com
}

Recibido: 10/04/06; aceptado: 20/10/06 biotransformación, así como cualquier alteración bioquímica precoz, cuya determinación en los fluidos biológicos, los tejidos o el aire exhalado evalúe la intensidad de la exposición al agente químico contaminante ambiental u ocupacional $(1,7)$. Para los plaguicidas inhibidores de la colinesterasa, es difícil contar con un indicador biológico de exposición basado en su determinación en sangre, pues tales indicadores se hidrolizan rápidamente. La medición de sus productos de biotransformación en orina tiene limitaciones, y su análisis aislado no evalúa la magnitud de la exposición. Entre los indicadores biológicos de efecto hasta ahora conocidos, los más importantes corresponden a la actividad colinesterásica y a la estearasa neurotóxica. La determinación de esta última en linfocitos y plaquetas es una prueba útil en los estudios de neurotoxicidad retardada. La medición de la actividad colinesterásica se ha constituido en la principal prueba de laboratorio para la vigilancia de la población laboral expuesta a estos plaguicidas (7). Específicamente para los plaguicidas inhibidores de la colinesterasa, que continúan siendo responsables de un alto número de intoxicaciones, la determinación de esta enzima se emplea como uno de los mejores indicadores biológicos de efecto en la exposición aguda o crónica (1). Existen varios métodos para la medición de esta enzima (8-18). Para la exposición prolongada en bajas dosis se recomienda medir la enzima eritrocitaria, mientras que para la exposición aguda se prefiere la medición de la enzima plasmática $(14,15)$.

También en Colombia los plaguicidas inhibidores de la colinesterasa son los responsables de la mayoría de intoxicaciones (herbicidas, fungicidas, 
insecticidas) (19-21). En América Latina, Colombia ocupa el tercer lugar (después de Brasil y México) en el consumo de plaguicidas (22) y viene en incremento tanto el consumo como la producción (23). En Centroamérica, la tasa de incidencia de intoxicaciones con estos plaguicidas era de 6,32 por cien mil habitantes en 1992 y progresivamente fue creciendo hasta llegar a 19,50 por cien mil en 2000 , o sea que se triplicó; la letalidad fue de 11\% en 2000 (Henao S. Situación epidemiológica de las intoxicaciones agudas con plaguicidas en el istmo centroamericano 1992-2000. Biomédica 2002;22(Suppl.1):39-40).

El programa colombiano de vigilancia epidemiológica de plaguicidas organofosforados y carbamatos opera desde 1981 y lo hace por un convenio entre el Laboratorio de Salud Ambiental del Instituto Nacional de Salud y las direcciones seccionales de salud del país; de 1993 a 1995 se encontró que el $6,2 \%$ de las 41.899 personas examinadas (expuestas a plaguicidas inhibidores de la colinesterasa o carbamatos) en 17 seccionales tenía resultados anormales de colinesterasas (22), cifra que fue similar $(6,1 \%)$ en el período 1996-1997 (23), pero hay zonas como los departamentos de Córdoba y Bolívar donde el porcentaje es muy alto, 17,7 y $20,3 \%$, respectivamente, lo que implica que uno de cada cinco a seis expuestos está intoxicado, situación más grave si se conoce que apenas el $36 \%$ de los estudiados tenía afiliación a la seguridad social (22). En el departamento del Meta, entre 1996 y 1997, de 1.184 personas con antecedentes de exposición a plaguicidas inhibidores de la colinesterasa, el 9\% mostró valores bajos anormales de actividad enzimática según medición con Lovibond ${ }^{\circledR}(24)$.

En Colombia, los valores de referencia de la colinesterasa se han tomado de estudios de países con características poblacionales, genéticas, biológicas, socioeconómicas y culturales muy diferentes del nuestro. Además, casi siempre tales estándares han surgido de estudios con grupos muy pequeños no representativos de la población. En 1990, Henao y colaboradores publicaron un informe sobre valores de colinesterasa en 400 menores trabajadores (14 a 17 años) no expuestos a estos plaguicidas (25). Nosotros realizamos en 19961997 un estudio en dos muestras representativas de sendas poblaciones laborales no expuestas a plaguicidas inhibidores de la colinesterasa residentes en el valle de Aburrá una (compuesta por 415 personas) y en el cercano oriente antioqueño la otra (412 personas), trabajo del cual han surgido datos sobre valores autóctonos de referencia en adultos para colinesterasa en sangre total (medida con la técnica Lovibond) (26), en eritrocitos (medida con los procedimientos de Michel y $\mathrm{EQM}^{\circledR}$ ) (27) y en plasma (medida con las técnicas de Michel, $\mathrm{EQM}^{\circledR}$ y Monotest $^{\circledR}$ ) (28); también se tienen modelos matemáticos para convertir los datos de una técnica plasmática en datos según otra (enviado para publicación). De igual manera, informamos del comportamiento de las colinesterasas eritrocitaria y plasmática en mujeres trabajadoras según su estado hormonal sexual (29), así como de valores de referencia de hemoglobina y hematocrito en población laboral (30). También se tienen valores de colinesterasas eritrocitaria y plasmática en trabajadores con enfermedades crónicas controladas y en usuarios de medicamentos (31).

El objetivo de este trabajo fue encontrar las relaciones cuantitativas (correlación y regresión) entre las mediciones de acetilcolinesterasa eritrocitaria según dos técnicas (Michel y $\mathrm{EQM}^{\circledR}$ ), con el fin de facilitar la conversión de las mediciones por un método a los valores correspondientes en el otro, pues en el país se usan procedimientos diferentes con fines epidemiológicos y clínicos. Los resultados sobre las variaciones de la enzima según región, sexo y edad se publicaron en otro artículo (27).

\section{Materiales y métodos}

\section{Diseño de la muestra poblacional, recolección de información, criterios de inclusión en el estudio}

Los detalles metodológicos del estudio se publicaron antes (26-31). Se usó un diseño descriptivo, transversal y prospectivo, para el cual se tomaron sendas muestras representativas de la población laboral adulta de 18 a 59 años de edad no expuesta a plaguicidas inhibidores de colinesterasa, vinculada a empresas afiliadas al 
Seguro Social y situadas en el Valle de Aburrá y en el cercano oriente antioqueño. Además, y sólo para fines complementarios, se incluyeron personas de 60 a 75 años.

Todos los trabajadores se captaron en la empresa en plena actividad laboral. Se investigó sobre la presencia de enfermedades que alteran los niveles de colinesterasas y se excluyó del estudio a quienes tuvieran alguna de ellas. Los estados fisiológicos como embarazo y menstruación, que modifican los valores de actividad colinesterásica, no fueron causa de exclusión, ni tampoco lo fue la declaración del trabajador de tener una enfermedad controlada; tampoco fue causa de exclusión la ingestión de medicamentos si el trabajador decía sentirse bien.

\section{Técnicas de colinesterasa eritrocitaria}

A cada trabajador se le realizaron mediciones de la actividad colinesterásica en eritrocitos mediante un método potenciométrico (Michel) y otro colorimétrico $\left(\mathrm{EQM}^{\circledR}\right)$. Las técnicas empleadas están basadas en los principios de: a) la medición del ácido producido de la acetilcolina, acetil betametilcolina o de la butirilcolina; b) la medición de la hidrólisis de la tiocolina (8-18).

El método potenciométrico de Michel mide la cantidad de ácido según el cambio de $\mathrm{pH}$ producido por la acción de la enzima en una solución tampón estándar durante un tiempo determinado. La unidad que emplea es delta de $\mathrm{pH} / \mathrm{hora}$. El método $\mathrm{EQM}^{\circledR}$ o método espectrofotométrico con colorímetro con fuente diódica emisora de luz (LESDC, por la sigla en inglés) mide la cantidad de ácido en función del cambio de color, el cual, a la vez, revela la modificación del $\mathrm{pH}$. Este método LEDSC aplicado hace uso de un fotocolorímetro portátil fabricado por EQM Research Inc. (Cincinnati, Ohio, USA); se utilizó el modelo 176. Esta prueba mide la oxihemoglobina presente en la muestra de sangre como un paso intermedio, y emplea la cifra de esta hemoglobina para ajustar el valor de la colinesterasa eritrocitaria. Los datos siempre deben usarse corregidos según el nivel de oxihemoglobina de la persona. La unidad empleada son las unidades por gramo de oxihemoglobina ( $\mathrm{U} / \mathrm{g}$ oxihemoglobina). Las características esenciales de las técnicas y métodos usados para medir la colinesterasa eritrocitaria se indican en el cuadro 1.

\section{Aspectos éticos}

El proyecto fue aprobado en todos sus aspectos por el Centro de Investigaciones de la Facultad Nacional de Salud Pública, Universidad de Antioquia (Medellín), y por el Fondo de Promoción de la Salud Industrial, Seguro Social (Bogotá). A los trabajadores, tanto grupal como individualmente, les fue explicada la investigación y cada uno firmó su consentimiento informado escrito.

\section{Análisis estadístico}

Se realizó un análisis de correlación y regresión lineal simple usando los programas SGPlus $7.1 \mathrm{y}$

Cuadro 1. Características esenciales de los métodos y técnicas para medir la colinesterasa eritrocitaria ${ }^{(1)}$.

\begin{tabular}{|c|c|c|}
\hline $\begin{array}{l}\text { Método } \\
\text { Y técnica }\end{array}$ & $\begin{array}{l}\text { Michel } \\
\text { (Electrométrica) }\end{array}$ & $\begin{array}{l}\mathrm{EQM}^{\circledR} \text { corregido o de Ellman modificado } \\
\text { (Espectrofotométrica; lectura a } 440 \mathrm{~nm} \text { ) }\end{array}$ \\
\hline Principio de análisis & Medición del pH & Colorimétrico cinético o de punto final \\
\hline Principio & Acetilcolina $-\mathrm{CE} \longrightarrow$ colina + acetato $+\mathrm{H}$ & $\begin{array}{l}\text { a) Propionilcolina }+\mathrm{H}_{2} \mathrm{O}-\mathrm{CE}->\text { propionato }+ \\
\text { tiocolina } \\
\text { b) Tiocolina + DNTB } \longrightarrow \text { ácido 5-tio-2- } \\
\text { nitrobenzoico + mezcla de disulfuros }\end{array}$ \\
\hline Muestra & Eritrocitos & Eritrocitos \\
\hline Tiempo de reacción & 60 minutos & 3 minutos \\
\hline Volumen & $20 \mu \mathrm{L}$ & $10 \mu \mathrm{L}$ \\
\hline Temperatura & $25^{\circ} \mathrm{C}$ & $21-40^{\circ} \mathrm{C}$ \\
\hline
\end{tabular}


SPSS 9.0. El análisis se efectuó con las dos variables correspondientes a las colinesterasas eritrocitarias medidas por Michel y EQM, que son de índole cuantitativa continua. Es necesario precisar que: a) es normal (gaussiana) la forma de cada variable cuantitativa $Y$ (cada conjunto de datos de cada prueba colinesterásica en eritrocitos), tal como se deduce de la estadística descriptiva, del histograma de frecuencias y de las pruebas para normalidad (pruebas de Kolmogorov-Smirnov y ji cudrado para bondad de ajuste); b) el tamaño de la muestra en cada estrato de edad es igual o mayor de 30 en tres grupos (menores de 30, de 30 a 39 y de 40 a 49 años) pero menor de 30 en los otros dos grupos (50 a 59 y 60 a 75 años). Por este motivo, es decir, tener unas muestras con tamaño menor de 30 , se optó por emplear la estadística t para contrastar el coeficiente b; c) la estadística t fue la empleada para la docimasia tanto de «r» como de «b». Dicha estadística la usa el programa estadístico para computador conocido como SPSS (Statistical Package for Social Sciences), para ambiente DOS, que fue el aplicado por nosotros para la dócima de b. Supone, entonces, para el caso de «b» que la variable $Y$ no se comporta como una normal o que la muestra es menor de $30 \mathrm{y}$, para el caso de $r$, supone que «r» poblacional es cero y que cada estrato por edad tuvo un tamaño igual o mayor que 30 . Mediante cálculos manuales hicimos el contraste de la hipótesis nula para $r$, según la cual « $r$ » es igual a cero, usando la prueba $t$ con ( $n-2)$ grados de libertad, y d) la decisión sobre significación estadística se tomó siempre con base en $p<0,05$.

\section{Resultados}

\section{Colinesterasa eritrocitaria según técnica, región y sexo}

A continuación se resumen los hallazgos.

1) Con la técnica de Michel, los valores mínimo y máximo fueron 0,564 y 1,207 deltas $\mathrm{pH} /$ hora. El promedio fue de 0,857 delta $\mathrm{pH} /$ hora con un intervalo de confianza del 95\% (IC95\%) de 0,849 a 0,866 (cuadro 2). La actividad enzimática difirió significativamente entre regiones (Aburrá y Oriente); dentro de cada región, la actividad enzimática masculina fue significativamente
Cuadro 2. Promedios e IC95\% (obtenidos por análisis de varianza de 3 vías) para las colinesterasas eritrocitarias según las técnicas de Michel (deltas $\mathrm{pH} /$ hora) y de $\mathrm{EQM}^{\circledR}$ corregida (U/g oxihemoglobina),

\begin{tabular}{lcccr}
\hline & n & Promedio & $\begin{array}{c}\text { Error } \\
\text { estándar }\end{array}$ & $\begin{array}{r}\text { IC95\% del } \\
\text { promedio }\end{array}$ \\
\hline A. Michel & 827 & 0,857 & 0,0044224 & 0,849 a 0,866 \\
B. EQM & 827 & 35,21 & 0,1994583 & 34,82 a 35,60 \\
\hline
\end{tabular}

mayor que la femenina; en ninguno de los estratos de región-sexo hubo influencia estadísticamente significativa de la edad en la actividad enzimática, cualquiera que fuera el método utilizado.

a) Aburrá: en las 415 personas, la media aritmética de la colinesterasa eritrocitaria fue 0,865 deltas $\mathrm{pH} /$ hora y el error estándar fue de 0,00643 , con un intervalo de confianza del 95\% (IC95\%), que osciló entre 0,849 y 0,866: hombres (193 personas): $0,877 \pm 0,00972$ (IC95\%: 0,858 a 0,896 ); mujeres (222 personas): 0,854 $\pm 0,00842$ (IC95\%: 0,837 a 0,870);

b) Oriente: en 412 personas, el promedio y el error estándar son $0,850 \pm 0,00607$, con IC95\% entre 0,838 y 0,862; hombres (197 individuos): 0,859 \pm 0,00880 (IC95\%: 0,842 a 0,876); mujeres (215 personas): 0,841 $\pm 0,00837$ (IC95\%: 0,824 a 0,857).

2) Con la técnica $E Q M^{\circledast}$, los valores mínimo y máximo fueron 19,1 y $57,7 \mathrm{U} / \mathrm{g}$ oxihemoglobina. El promedio fue de $35,21 \mathrm{U} / \mathrm{g}$ oxihemoglobina con IC95\% de 34,82 a 35,60. Con la técnica $E M^{\circledR}$ no hay diferencia estadísticamente significativa entre regiones, ni entre sexos ni entre grupos de edad.

a) Aburrá: en las 415 personas, el promedio y el error estándar fueron 35,17 $\pm 0,28996$ (IC95\%: $34,60$ a 35,74$)$; hombres: $35,35 \pm 0,43819$ (IC95\%: 34,49 a 36,21); mujeres: 34,99 \pm 0,37987 (IC95\%: 34,25 a 35,74).

b) Oriente: en 412 personas, el promedio y el error estándar fueron 35,26 \pm 0,27396 (IC95\%: 34,72 a 35,79); hombres: 34,86 \pm 0,39703 (IC95\%: 34,08 a 35,64); mujeres: 35,66 \pm 0,37762 (IC95\%: 34,91 a 36,40$)$.

Correlación-regresión entre técnicas de Michel y EQM (incluidos datos extremos). En la población 
total $(n=827)$ de 827 personas estudiadas, sin tener en cuenta las variaciones existentes entre regiones y entre sexos,que como ya se anotó en realidad existen en el caso de la técnica de Michel, y que posiblemente se deben a que en esta técnica los valores de la enzima no están corregidos según el valor de hemoglobina, el coeficiente $r$ vale 0,666 y $R^{2}$ es de 44,30; las ecuaciones de regresión lineal de una prueba en otra son: EQM U/g oxihemoglobina $=9,57509 \mathrm{U} / \mathrm{g}$ oxihemoglobina + 29,7914 (Michel deltas de $\mathrm{pH} /$ hora); Michel deltas de $\mathrm{pH} /$ hora $=0,33120$ deltas de $\mathrm{pH} /$ hora $+0,01487$ (EQM U/g oxihemoglobina) (figura 1 ).

El análisis de varianza asociado a estas ecuaciones generales (para las 827 personas) indica que los coeficientes «a» (intersección) y «b» (pendiente) son altamente significativos $(p=$ 0,00000).

Estratos de región y de sexo. Una síntesis del análisis detallado de correlación y regresión lineales para $\mathrm{EQM}^{\circledR}$ y Michel teniendo en cuenta las diferencias por región y por sexo muestra que tanto en Aburrá como Oriente, y en uno como en otro sexo (cuadro 3), el valor del estadístico $t$ asociado a la docimasia para «a» fue superior a 2,0 (en cifras absolutas, sin importar el signo), lo cual implica que el valor de «a» es estadísticamente diferente de cero y que, por tanto, «a» influye en el comportamiento del modelo; hay correlación positiva $(r=0,635$ a $0,770)$. Hubo mayores valores de «r» en Aburrá que en Oriente; en Aburrá, la correlación es mayor entre los hombres que entre las mujeres, pero en Oriente es al contrario. Este análisis de los estratos región-sexo demostró que los cambios del coeficiente "r" son de poca magnitud con relación al grupo total (sin estratificar) y, por ello, se decidió trabajar con el grupo de 827 trabajadores $(r=0,666)$; el valor de $R^{2}$ oscila, en consecuencia, entre $40 \%$ (hombres de Oriente) y $59 \%$ (hombres de Aburrá), lo cual significa que 40 a $59 \%$ de la variación del Michel se explica por la variación en el EQM ${ }^{\circledR}$ y viceversa; el valor del coeficiente «b» (pendiente) es positivo y el valor de la prueba t asociada es mayor que 2,0 en cifras absolutas, lo que indica que el valor del coeficiente $« b »$ muestral tiene diferencia

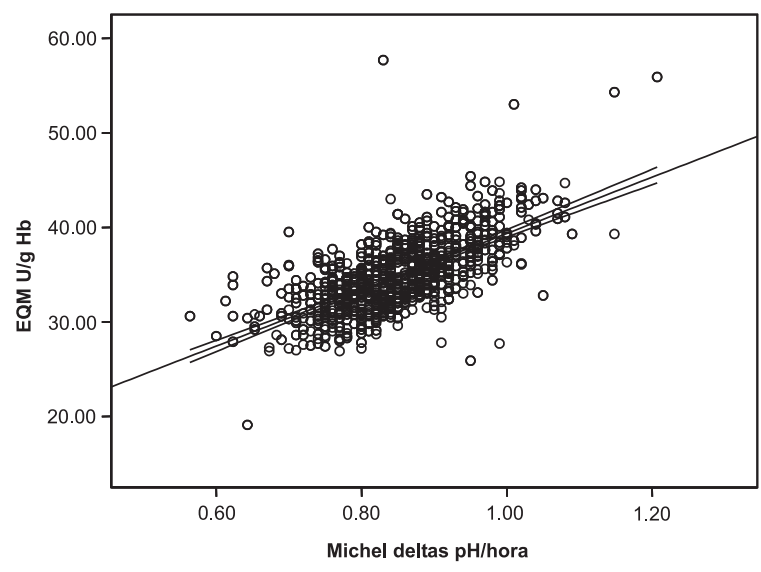

Figura 1. Correlación-regresión entre técnicas de Michel y EQM para acetilcolinesterasa (incluye datos extremos; $\mathrm{n}=827$ )

estadísticamente significativa con respecto al coeficiente poblacional, es decir que no puede aceptarse la hipótesis nula de que $b=0 \mathrm{y}$, por ello, deberá aceptarse que la variación de $\mathrm{EQM}^{\circledR}$ (variable $Y$ ) se ve afectada por la variación de Michel (variable $X$ ), o viceversa (Michel en $\mathrm{EQM}^{\circledR}$ ). Comparado con Oriente, en Aburrá el EQM ${ }^{\circledR}$ cambia más por cada unidad que lo que varía el Michel.

Correlación-regresión entre técnicas de Michel y EQM (excluidos datos extremos). Para el grupo de 827 personas estudiadas, es decir, sin considerar las variaciones existentes entre regiones y entre sexos, el coeficiente $r$ puede elevarse de 0,66558 a más de 0,718 al eliminar doce de los valores extremos (outliers, en inglés), es decir, los valores que se encuentran por fuera de las bandas de confianza de la ecuación de regresión y que influyen marcadamente en el valor de r. Los valores extremos son más de doce, pero basta excluir esos doce para obtener esa mejoría en el coeficiente r. De los doce datos, ocho son extremos tanto en la regresión de Michel en EQM ${ }^{\circledR}$ como en la $\mathrm{EQM}^{\circledR}$ en Michel, mientras los otros cuatro difieren de una regresión a la otra. Las ecuaciones ajustadas (excluidos los valores extremos) son: EQM U/g oxihemoglobina = $8,18840 \mathrm{U} / \mathrm{g}$ oxihemoglobina $+31,3920$ (Michel deltas de $\mathrm{pH} /$ hora); Michel deltas de $\mathrm{pH} /$ hora $=$ 0,292510 deltas de $\mathrm{pH} /$ hora $+0,016101$ (EQM U/ g oxihemoglobina). 
Cuadro 3. Correlación y regresión lineales simples entre las colinesterasas eritrocitarias medidas con las técnicas $E Q M^{\circledR}$ y de Michel según región y sexo.

A. Valores de los coeficientes de correlación «r» y de determinación $\mathrm{R}^{2}$ para $E Q \mathrm{M}^{\circledast}$ en Michel y viceversa

\begin{tabular}{lllll}
\hline Región & Sexo & $\mathbf{n}^{(1)}$ & $\mathbf{r}^{(1)}$ & $\mathbf{R}^{\mathbf{2}}(\%)$ \\
\hline Aburrá & & & & \\
& Hombres & 193 & 0,770 & 59 \\
\multirow{2}{*}{ Oriente } & Mujeres & 222 & 0,715 & 51 \\
& Hombres & 197 & 0,635 & 40 \\
& Mujeres & 215 & 0,684 & 47 \\
\hline
\end{tabular}

(1) En todos los casos, el valor de $p$ asociado a la estadística t para el coeficiente $r$ es menor de 0,001 (uno por mil) para $(n-2)$ grados de libertad, lo que implica que hay una diferencia altamente significativa entre el valor de «r» y cero. Recuérdese que si el valor de « $r »$ es estadísticamente significativo a un nivel dado, por ejemplo $5 \%(\alpha=0,05)$, entonces «b» será igualmente significativo a ese mismo nivel.

(2) La cifra se refiere al tamaño del estrato por región y sexo.

B. Ecuaciones de regresión lineal entre $E Q M^{\circledR}$ y Michel (modelo $Y=a+b X)$

Datos sin ajustar

EQM U/g oxihemoglobina $=9,57509 \mathrm{U} / \mathrm{g}$ oxihemoglobina + 29,7914 (Michel deltas de $\mathrm{pH} /$ hora); Michel deltas de $\mathrm{pH} /$ hora $=0,33120$ deltas de $\mathrm{pH} /$ hora $+0,01487(E Q M ~ U / g$ oxihemoglobina).

Datos ajustados (12 valores extremos excluidos en cada ecuación, ocho de ellos comunes a ambas).

EQM U/g oxihemoglobina $=8,18840 \mathrm{U} / \mathrm{g}$ oxihemoglobina + 31,3920 (Michel deltas de $\mathrm{pH} /$ hora); Michel deltas de $\mathrm{pH} /$ hora $=0,292510$ deltas de $\mathrm{pH} /$ hora $+0,016101(\mathrm{EQM} \mathrm{U} / \mathrm{g}$ oxihemoglobina).

Nota: con los datos sin ajustar o ajustados, los coeficientes a (intersección) y b (pendiente) son estadísticamente significativos $(p=0,00000)$ en el modelo.

Con las ecuaciones ajustadas se conservan todas las asociaciones y valores estadísticamente significativos hallados con los datos sin ajustar. Por supuesto, se recomienda usar las ecuaciones ajustadas, con las que el coeficiente $\mathrm{R}^{2}$ alcanza a valer al menos $51 \%$.

Como dato adicional, cabe anotar que las pruebas eritrocitarias en función de las plasmáticas tienen una muy baja, casi nula, correlación, pues tanto en Aburrá como en Oriente, y en uno y otro sexo, la correlación que a veces es positiva y otras negativa tiene siempre $r<0,230$, y excepto en dos casos, siempre $r<0,120$.

\section{Discusión}

Para que la conversión de unidades de las técnicas de medición de colinesterasa eritrocitaria sea adecuada se requiere que los dos métodos se relacionen entre sí mediante un modelo matemático que exprese en forma adecuada esa asociación; en este informe se da cuenta del resultado del análisis de tal asociación mediante el procedimiento de regresión lineal. La regresión lineal es un modelo aceptable para medir el comportamiento de una prueba colinesterásica en función de otra, pues en las 827 personas, las pruebas cuantitativas eritrocitarias (Michel, $E Q M \circledR)$ tienen una correlación fuertemente significativa, la cual es posible mejorar al eliminar el $1,5 \%$ ( 12 de 827 datos $X Y$ ) de valores extremos (outliers) cuando se efectúa cada regresión. La correlación lineal $(r=0,666$ para datos sin ajustar y $r=0,718$ tras eliminar $1,5 \%$ de datos extremos) es explicable porque los datos corresponden a mediciones reales de campo y no a trabajo de laboratorio in vitro. La asociación entre las dos variables siempre es altamente significativa y tanto la intersección como la pendiente influyen en el modelo de manera significativa, bien sea con los datos brutos o con los ajustados. El ajuste de los datos es un procedimiento apropiado y permite mejorar notoriamente la correlación entre las variables. Las predicciones deberán hacerse en función de los datos mínimo y máximo observados, que se indicaron antes (mínimo y máximo de Michel: 0,564 y 1,207 deltas pH/hora; de EQM: 19,1 y $57,7 \mathrm{U} / \mathrm{g}$ oxihemoglobina).

Es indispensable considerar que los modelos matemáticos descritos para la conversión son valederos en las condiciones en que se midió la actividad enzimática; si tales condiciones cambian también habrá una variación en el comportamiento de tal actividad $y$, en consecuencia, en los datos obtenidos, lo cual llevará a una modificación de los modelos o ecuaciones. El método electrométrico aplicado por Michel (10) y consolidado por Rider y asociados (12) mide el pH y es la prueba de referencia o estándar para cuantificar la actividad colinesterásica en eritrocitos y plasma $(1,2)$; requiere condiciones de laboratorio y personal bien entrenado. El método espectrofométrico usado por 
Ellman y aplicado por la técnica de $\mathrm{EQM}^{\circledR}$ es un procedimiento colorimétrico, cinético o de punto final, simple, que no requiere un laboratorista con mucha destreza; es rápido, conveniente y confiable, tiene muy buena precisión y óptima sensibilidad, es más rápido y sensible que Michel y la versión desarrollada en 1978 por la Organización Mundial de la Salud con mini-espectrofotómetro portátil sirve para trabajo de campo y mantiene todas las ventajas enunciadas (13-17).

Da Silva y Midio informaron sobre conversión de unidades de tres métodos para medir colinesterasas en sangre (métodos de Ellman para sangre total y plasma, de Michel para plasma y eritrocitos, de Edson para sangre total) (32); también se conoce el informe de Newman y Que Hee sobre conversión de resultados de tres técnicas para colinesterasa en plasma (técnica con analizador de Du Pont, técnica «ChE-tel» de Pfizer y técnica de Michel) (33). Otros informes conocidos son el de Groff, Kaminskis y Hellin, quienes informaron sobre la conversión de unidades de actividad enzimática colinesterásica medida por técnica manual de Michel y automática (34); el de Morse, centrado en la conversión de mediciones de colinesterasa con las técnicas de Michel y de Garry \& Routh (técnica de DTNB) (35), y el de Grainger y asociados sobre correlación entre valores obtenidos con una técnica manual y una automatizada (36). Estos informes señalan la posibilidad y la conveniencia de disponer de estos modelos para efectuar tales intercambios, lo cual debe hacerse para condiciones específicas de laboratorio, es decir, teniendo en cuenta las condiciones aplicadas al realizar la medición química.

Hay varios factores que contribuyen a la variación de los valores enzimáticos; entre los presentes en esta investigación se encuentran la técnica química (Michel, EQM ${ }^{\circledR}$ ), la región (valle de Aburrá y cercano oriente antioqueño) y el sexo. La actividad de la colinesterasa eritrocitaria fue estadísticamente igual por región con la técnica $\mathrm{EQM}^{\circledR}$, pero con la técnica de Michel la media aritmética fue estadísticamente mayor en Aburrá que en Oriente (0,865 vs. 0,850 delta de $\mathrm{pH} /$ hora). En cada región, los hombres presentaron actividad enzimática estadísticamente mayor que las mujeres con cada una de las técnicas. En cada estrato región-sexo, la edad no influyó en la actividad.

El sistema de conversión de unidades de colinesterasa eritrocitaria aquí descrito ayudará en las actividades de atención clínica y de vigilancia epidemiológica, en especial a personas expuestas, por razones laborales, accidentales o voluntarias, a plaguicidas inhibidores de la colinesterasa, pero también a otros individuos en quienes la medición de la enzima plasmática es frecuente (enfermedades hepáticas, renales y neurológicas, sarcoidosis). La frecuencia y gravedad de las intoxicaciones por plaguicidas inhibidores de la colinesterasa indican que el problema de las intoxicaciones está vigente; así, por ejemplo en Colombia, en el departamento del Valle, hubo 367 intoxicaciones agudas en 1997, de las cuales el $41 \%$ fue por exposición ocupacional, $33 \%$, voluntaria, $11 \%$, accidental y $14 \%$, sin dato (19). En la ciudad de Pasto, capital del departamento de Nariño, en 1997 ocurrió una intoxicación con harina contaminada con paratión proveniente de Ecuador, que afectó a más de 300 personas y causó 120 hospitalizaciones y 15 muertes (20). En el departamento de Antioquia, entre 1998 y 2001 se presentaron 463 muertes por intoxicaciones agudas por diversas sustancias, incluidos los plaguicidas inhibidores de la colinesterasa, que explicaban la casi totalidad del $20 \%$ debido a exposición laboral; entre 2000 y 2002 los casos aumentaron en $70 \%$ (la incidencia pasó de 2,8 a 3,9 por cien mil habitantes) (21). Como ya se anotó, en el departamento del Meta, en 1996-1997, 9\% de 1.184 personas expuestas a estos plaguicidas mostró valores anormalmente bajos de actividad enzimática (24); en esa región también hay fumigaciones masivas con herbicidas como paraquat y glifosato (37). En Colombia, de 1993 a 1997 se examinaron 70.357 sujetos expuestos a plaguicidas inhibidores de la colinesterasa o los carbamatos y el porcentaje de personas con valores bajos de colinesterasa plasmática (medida por Lovibond) fue de $5,4 \%$ en 1993, subió a $6,5 \%$ en 1994 , fue de $6,0 \%$ en 1995 , de $7,4 \%$ en 1996 y de $5,2 \%$ en $1997(22,23,38)$. La importancia de disponer en forma ágil y precisa de un sistema de conversión de unidades de colinesterasa eritrocitaria se capta a partir de estos 
ejemplos, que ponen de manifiesto la necesidad de ese sistema, tanto en el ámbito clínico (diagnóstico, gravedad o intensidad de la intoxicación, seguimiento de la evolución), como en el terreno epidemiológico (evaluación periódica de los trabajadores expuestos, encuestas).

\section{Financiación}

Esta investigación fue financiada por el Fondo de Promoción de la Salud Industrial, Seguro Social (Colombia) y la Universidad de Antioquia.

\section{Conflictos de interés}

Ninguno conocido por el autor.

\section{Referencias}

1. Henao S, Corey G. Plaguicidas inhibidores de las colinesterasas. Serie Vigilancia 11. Centro Panamericano de Ecología Humana y Salud. Metepec: ECO, OPS, OMS; 1991.

2. International Programme on Chemical Safety (IPCS). Organophosphorous insecticides: a general introduction. Environmental Health Criteria № 63 . Geneve: WHO, ILO; 1986.

3. International Programme on Chemical Safety (IPCS). Carbamate pesticides: a general introduction. . Environmental Health Criteria № 64. Geneve: IPCS; 1986.

4. Whittaker $\mathbf{M}$. Cholinesterase. Monographs in Human Genetics, № 11. New York: Karger Basel; 1986.

5. Guyton AK. Tratado de fisiología médica. 10 ed. México, D.F.: Interamericana-Mc Graw Hill; 2000.

6. Schwarz M, Glick D, Lowenstein Y, Soreq H. Engineering of human cholinesterases explains and predicts diverse consequences of administration of various drugs and poisons. Pharmacol Ther 1995;67:283322.

7. Carlock LL, Chen WL, Gordon EB, Killeen JC, Manley A, Meyer LS, et al. Regulating and assessing risks of cholinesterase-inhibiting pesticides: divergent approaches and interpretations. J Toxicol Environ Health B Crit Rev 1999;2:105-60.

8. Aldridge WN. The nature of the reaction of organophosphorus compounds and carbamates with esterases. Bull World Health Organ 1971;44:25-30.

9. Kangas J, Jauhiainen A. Determination of cholinesterase activity. Afr Newslett Occup Health Safety 1991;2:56-8.

10. Michel HO. An electrometric method for determination of red blood cell and plasma cholinesterase activity. J Lab Clin Med 1949;34:1564-8.
11. Nabb DP, Whitfield F. Determination of cholinesterase by the automated delta $\mathrm{pH}$ stat method. Arch Environ Health 1967;15:147-54.

12. Rider JA, Hodges JL Jr, Swader J, Wiggins AD. Plasma and cell cholinesterase in 800 "healthy" blood donors. J Lab Clin Med 1957;50:376-83.

13. Ellman GL, Courtney KD, Andres V, Featherstone RM. A new rapid colorimetric determination of acetylcholinesterase activity. Biochem Pharmacol 1961;7:8895.

14. Magnotti RA Jr, Eberly JP, Quarm DE, McConell RS. Measurement of acetylcholinesterase in erythrocytes in the field. Clin Chem 1987;33:1731-5.

15. Magnotti RA Jr, Dowling K, Eberly JP, McConell RS. Field measurement of plasma and erythrocytes cholinesterases. Clin Chem Acta. 1988;176:315-32.

16. Riddles PW, Blakeley RL, Zerner B. Reassessment of Ellman's reagent. Methods Enzymol 1983;91:49-60.

17. EQM Research Inc. Cholinesterase kit for the field determination of pesticide exposure. Instruction manual. Cincinnati: EQM; 1994.

18. Tintometer. Lovibond. The Lovibond cholinesterase test kit AF 267 (40-2670). Instruction manual. Virginia, USA: Tintometer; sin fecha.

19. Morales L, Silva E, Ortiz JE, Ramírez P, García A. Intoxicación por plaguicidas en el departamento del Valle del Cauca, 1997. Inf Quinc Epidem Nac 1998;3:222-5.

20. Idrobo AJ. Intoxicaciones masivas con plaguicidas en Colombia. Biomédica 1999;19:67-76.

21. Henao GL, Bolívar JA, Orozco RE. Análisis de las intoxicaciones en el departamento de Antioquia. Inf Quinc Epidem Nac 2003;8:305-20.

22. Varona M, Morales L, Ortiz J, Sánchez J, Cárdenas O, De la Hoz F. Panorama epidemiológico de exposición a plaguicidas inhibidores de colinesterasa en 17 departamentos del país. Biomédica 1998; 18:22-9.

23. Silva E, Morales L, Ortiz JE. Evaluación epidemiológica de plaguicidas inhibidores de acetilcolinestersas en Colombia, 1996-1997. Biomédica 2000;20:200-9.

24. Silva E, Morales L, Ortiz JE, Díaz ML. Evaluación epidemiológica de plaguicidas en el departamento del Meta. Programa VEO, 1996 a 19997. Inf Quinc Epidem Nac 2002;7:389-401.

25. Henao S, Zapata FM, Restrepo MP, Marín LE, Ramírez H, Corrales R, et al. Actividad colinesterásica en menores trabajadores. Antioquia (Colombia), 19891990. Medellín: Seguro Social, Universidad de Antioquia; 1990. p.115.

26. Carmona-Fonseca J, Henao S, Garcés R. Valores de referencia de actividad colinesterásica sanguínea en 
población laboral activa no expuesta a plaguicidas inhibidores de colinesterasa. Rev Fac Nal Salud Publ (Medellín) 2000;18:55-72.

27. Carmona-Fonseca J. Valores de referencia de la actividad de la colinesterasa eritrocitaria según las técnicas de Michel y EQM ${ }^{\circledR}$ en población laboral de Antioquia, Colombia. Rev Panam Salud Pública 2003;14:316-24.

28 Carmona-Fonseca J. Valores de referencia de colinesterasa plasmática con los métodos de Michel, $\mathrm{EQM}^{\circledR}$ y Monotest ${ }^{\circledR}$ en población laboral activa del departamento de Antioquia, Colombia. Biomédica 2003;23:437-55.

29. Carmona-Fonseca J. Valores de colinesterasas en trabajadores activas embarazadas, menstruantes, usuarias de anticonceptivos o menopáusicas. Rev Col Obstet Ginecol 2003;54:146-56.

30. Carmona-Fonseca J. Valores de referencia de hemoglobina y hematocrito en una población laboral colombiana. Acta Med Colom 2003;28:63-70.

31. Carmona-Fonseca J. Colinesterasas eritrocitaria y plasmática en trabajadores con enfermedades crónicas controladas y en usuarios de medicamentos. Iatreia 2006;19:14-28
32. Da Silva ES, Midio AF. Interconversion of units of three methods for blood cholinesterases. Med Lav 1998;89:265-72.

33. Newman MA, Que Hee SS. Interconversion and comparison of the results of three methods for cholinesterase in serum. Clin Chem 1984;30:308-10.

34. Groff WA, Kaminskis A, Ellin RI. Interconversion of cholinesterase enzyme activity units by the manual delta $\mathrm{pH}$ method and a recommended automated method. Clin Toxicol 1976;9:353-8.

35. Morse CS. Interconversion of cholinesterase measurements by the deltapH method of Michel and the DTNB method of Garry and Routh. Clin Toxicol 1974;7:38993.

36. Grainger MM, Groff WA, Ellin RI, Arsenal E. Blood cholinesterase values. Correlation obtained by automated and manual techniques. Arch Environ Health 1968;16:821-2.

37. Altamar-Rios J. Herbicidas y malformaciones congénitas en Meta (Colombia). An Otorrinolaringol Ibero Am 2002;29:1-20.

38. Sánchez JF, Morales L, Varona M, Camacho M, Ortiz J, De La Hoz F. Exposición a plaguicidas inhibidores de colinesterasa en 17 departamentos. Inf Quinc Epidem Nac 1997;2:10-12. 